\title{
Lactobacillus plantarum CUL66 can impact cholesterol homeostasis in Caco-2 enterocytes
}

\author{
D.R. Michael ${ }^{1}$, J.W.E. Moss ${ }^{2}$, D. Lama Calvente ${ }^{1}$, I. Garaiova ${ }^{1}$, S.F. Plummer ${ }^{1}$ and D.P. Ramji ${ }^{2}$ \\ ${ }^{1}$ Cultech Limited, Unit 2 Christchurch Road, Baglan Industrial Park, Port Talbot SA12 7BZ, United Kingdom; ${ }^{2}$ Cardiff \\ School of Biosciences, Cardiff University, Sir Martin Evans Building, Museum Avenue, Cardiff CF10 3AX, United Kingdom; \\ darynm@cultech.co.uk
}

Received: 8 October 2015 / Accepted: 23 December 2015

(c) 2016 Wageningen Academic Publishers

\section{OPEN ACCESS @(1)(- RESEARCH ARTICLE}

\begin{abstract}
Hypercholesterolemia drives the development of cardiovascular disease, the leading cause of mortality in western society. Supplementation with probiotics that interfere with cholesterol metabolism may provide a contribution to disease prevention. Lactobacillus plantarum CUL66 (NCIMB 30280) has been assessed in vitro for its ability to impact cholesterol absorption. L. plantarum CUL66 tested positive for bile salt hydrolase activity and the ability to assimilate cholesterol from culture media. RT-qPCR analysis showed that the bacterium significantly decreased the expression of Niemann-Pick C1-like 1 and ATP-binding cassette transporter-1 in polarised Caco- 2 cells after $6 \mathrm{~h}$ exposure. Conversely, the expression of ATP-binding cassette sub-family G member (ABCG)-5 and ABCG-8, and 3-hydroxy-3-methylglutaryl-CoA reductase were significantly increased. Using a radiolabelled assay, we also observed significant reductions in the uptake and basolateral efflux of cholesterol by Caco- 2 cells exposed to L. plantarum CUL66. This in vitro study identified L. plantarum CUL66 as a cholesterol lowering bacteria by highlighting its ability to beneficially regulate multiple in vitro events associated with intestinal cholesterol metabolism and provides evidence of efficacy for its inclusion in future in vivo studies.
\end{abstract}

Keywords: hypercholesterolemia, enterocyte, L. plantarum, uptake, efflux

\section{Introduction}

Hypercholesterolemia is one of the major risk factors driving the development of cardiovascular disease (CVD) which accounts for more global mortalities than any other ailment (WHO, 2015) and costs UK healthcare systems more than $£ 8.6$ billion per annum (Townsend et al., 2012). One of the most effective disease prevention approaches currently available is the routine use of statins which specifically inhibit the de novo synthesis of cholesterol (Buckley and Ramji, 2015; McLaren et al., 2011; Michael et al., 2012; Taylor et al., 2013). However, adverse side effects (Banach et al., 2015) and only an overall 25\% reduction in fatal and non-fatal CVD-related events (Taylor et al., 2013) indicate the need for additional approaches to reduce the burden of CVD. The lack of evidence correlating cholesterol consumption with serum cholesterol levels (Christie, 2015) has raised questions about the validity of a cholesterol restricted diet as a preventative measure suggesting that lifestyle changes alone cannot reduce susceptibility to the development of CVD. There is now growing evidence that some probiotic organisms (components of the indigenous microbiota) have the capacity to impact multiple aspects of the cholesterol metabolism of the host (Gorenjak et al., 2014; Huang and Zheng, 2010; Huang et al., 2010, 2013; Pereira and Gibson, 2002a,b; Yoon et al., 2011, 2013) resulting in reduced serum cholesterol levels (Fuentes et al., 2013; Mann, 1974; Pereira and Gibson, 2002b; Rerksuppaphol and Rerksuppaphol, 2015) and positive CVD-related outcomes (Sun and Buys, 2015).

A complex symbiotic relationship exists between the host's intestinal epithelium and the resident microbiota. The cholesterol lowering ability of some probiotics, which are defined as 'live organisms which when administered in adequate amounts confer a health benefit to the host' (FAO/ 
WHO, 2006), can be attributed to bile salt hydrolase (BSH) activity and/or the ability to assimilate cholesterol, that allows them to promote the removal of cholesterol from the intestinal lumen (Kumar et al., 2012). It is also emerging from numerous in vitro and in vivo studies that probiotics, including strains of Lactobacillus plantarum, can impart cholesterol lowering effects by modulating key mechanisms responsible for cholesterol absorption in the intestines of the host (Gorenjak et al., 2014; Huang and Zheng, 2010; Huang et al., 2013; Yoon et al., 2013).

Niemann-Pick C1-like 1 (NPC1L1) is a cholesterol transporter critical for the uptake of cholesterol and is highly expressed on the brush border of intestinal epithelial cells (Altmann et al., 2004). Disruption of NPC1L1 expression in genetically modified mice renders them almost completely resistant to diet induced hypercholesterolemia (Davis et al., 2007) and pharmacological inhibition of NPC1L1 function using ezetimibe is an effective strategy for the reduction of serum cholesterol level in humans (Cannon et al., 2015; Sudhop et al., 2002). ATP-binding cassette sub-family G member (ABCG)-5 and ABCG-8 are also key cholesterol transporters that are highly expressed on the apical surface of intestinal epithelial cells and mediate the efflux of intracellular cholesterol to bile salts back into the intestinal lumen (Tachibana et al., 2007; Vrins et al., 2007). Abolishment of ABCG-5/8 gene expression in mice results in the rapid accumulation of plasma cholesterol (Yu et al., 2002) and functional mutations of these genes cause sitosterolemia; a genetic disorder characterised by the accumulation of sterols in blood and tissues (Berge et al., 2000). ATP-binding cassette transporter-1 (ABCA-1) is also involved in the efflux of intracellular cholesterol from intestinal epithelial cells but is predominantly expressed on the basolateral membrane and preferentially utilises apolipoprotein-AI (Apo-AI) as a cholesterol acceptor (Ohama et al., 2002; Tachibana et al., 2007). Genetic deletion of ABCA-1 expression in mice results in reduced absorption of dietary cholesterol from the intestine (Drobnik et al., 2001).

In this study, the cholesterol lowering abilities of the strain L. plantarum CUL66 (NCIMB 30280) isolated from a healthy human have been determined and a more detailed assessment of the molecular mechanisms revealed its ability to reduce cholesterol uptake in an in vitro model of the intestinal epithelium.

\section{Materials and methods}

\section{Reagents}

All chemicals were purchased from Sigma-Aldrich (Poole, UK) unless otherwise stated.

\section{Bacterial strain and growth condition}

Bacterial isolate L. plantarum CUL66 (NCIMB 30280) was stored at $-20^{\circ} \mathrm{C}$ on Cryo-beads (Pro-Lab Diagnostics, Wirral, UK) until use and initial cultures were grown in De Man-Rogosa-Sharpe (MRS) broth (Oxoid, Basingstoke, UK) for $18 \mathrm{~h}$ at $37{ }^{\circ} \mathrm{C}$ under anaerobic conditions ( $10 \%$ carbon dioxide, $10 \%$ hydrogen, $80 \%$ nitrogen).

\section{Bile salt hydrolase activity}

BSH activity was tested using a modified plate assay. Briefly, MRS agar plates or MRS agar plates supplemented with $0.5 \%(\mathrm{w} / \mathrm{v})$ taurodeoxycholic acid (TDCA) were pre-reduced by incubation under anaerobic conditions at $37^{\circ} \mathrm{C}$ for $24 \mathrm{~h}$. Filter discs $(6 \mathrm{~mm})$ present on the agar surface were then inoculated with approximately $10^{7} \mathrm{cfu} / \mathrm{ml}$ L. plantarum CUL66. BSH activity was indicated by the development of a white precipitate around or on the colonies after $48 \mathrm{~h}$ incubation under anaerobic conditions at $37^{\circ} \mathrm{C}$. MRS agar plates lacking TDCA were also inoculated and served as negative controls in each experiment.

\section{Cholesterol assimilation}

The ability of L. plantarum CUL66 to assimilate cholesterol was assessed according to a modified version of a method described elsewhere (Pereira and Gibson, 2002a). Ten ml aliquots of sterile MRS broth supplemented with $0.3 \%(\mathrm{w} / \mathrm{v})$ ox-bile and $1 \mathrm{mg} / \mathrm{ml}$ polyoxyethanyl-cholesteryl sebacate (delivering approximately $200 \mu \mathrm{g} / \mathrm{ml}$ cholesterol) were left uninoculated (control) or inoculated with approximately $10^{8} \mathrm{cfu} / \mathrm{ml} \mathrm{L}$. plantarum CUL66. All cultures were then incubated for $18 \mathrm{~h}$ at $37^{\circ} \mathrm{C}$ under anaerobic conditions. Following incubation, bacterial cells were removed by centrifugation $(1000 \times g, 20 \mathrm{~min})$ and the culture supernatant was assayed for cholesterol content. In addition, the dry weight of the remaining bacterial pellets were determined following $2 \mathrm{~h}$ drying at $100^{\circ} \mathrm{C}$ and the amount of cholesterol removed expressed as mg of cholesterol removed (compared to the uninoculated control) per g of bacteria (dry weight).

\section{Cholesterol assay}

Total lipid was extracted from the culture supernatant and assayed for cholesterol content according to a modified version of a previously described method by Rudel and Morris (1973). Briefly, $2 \mathrm{ml}$ of pure ethanol and $2 \mathrm{ml}$ of $33 \%$ $(\mathrm{w} / \mathrm{v})$ potassium hydroxide were added to a $1 \mathrm{ml}$ sample of culture supernatant and mixed thoroughly. Samples were then incubated at $37^{\circ} \mathrm{C}$ for $15 \mathrm{~min}$ and cooled before $2 \mathrm{ml}$ deionised water and $3 \mathrm{ml} \mathrm{n}$-hexane were added and the mixing repeated. Samples were then incubated at ambient temperature for $15 \mathrm{~min}$ to allow phase separation before a $1 \mathrm{ml}$ sample of the upper clear phase was removed into a clean tube for rapid evaporation under nitrogen. Two 
$\mathrm{ml}$ of $o$-phthaldaldehyde solution $(0.5 \mathrm{mg} / \mathrm{ml}$ in glacial acetic acid) was then added to each tube and $0.5 \mathrm{ml}$ of concentrated sulphuric acid was then carefully added by slow pipetting down the side of the tube. Each sample was then mixed thoroughly and incubated for a further $10 \mathrm{~min}$ at ambient temperature. The absorbance of each sample was read at $550 \mathrm{~nm}$ using a UV-V spectrophotometer (Agilent Technologies, Santa Clara, CA, USA) and cholesterol concentration was determined by comparison with a standard curve composed of identically processed samples of known cholesterol concentration $(0,31.25,62.5,125,250$ and $500 \mu \mathrm{g} / \mathrm{ml}, \mathrm{R}^{2}=0.9975$ ).

\section{Caco-2 cell culture}

Caco-2 cells were obtained from American Type Culture Collection (ATCC, Middlesex, UK) and grown in Dulbecco's Modified Eagle's medium (DMEM) supplemented with $4,500 \mathrm{mg} / \mathrm{l}$ glucose, $1 \%(\mathrm{v} / \mathrm{v})$ non-essential amino acids, $10 \%$ (v/v) heat inactivated foetal bovine serum (Labtech, Sussex, $\mathrm{UK})$, penicillin $(100 \mathrm{U} / \mathrm{ml})$ and streptomycin $(100 \mathrm{U} / \mathrm{ml})$ at $37{ }^{\circ} \mathrm{C}$ in $5 \% \mathrm{CO}_{2}$ and $95 \%$ humidity. Caco-2 cells were seeded at $5 \times 10^{5}$ cells $/ \mathrm{cm}^{2}$ into standard tissue culture coated 24 well plates (Costar, Cambridge, UK) for realtime quantitative polymerase chain reaction (RT-qPCR) analysis and cholesterol uptake assays or polycarbonate semi-permeable transwell membranes $(0.4 \mu \mathrm{M}$ pores; Costar, Cambridge, UK) for cholesterol efflux assays. Caco- 2 cells were maintained for 18 to 21 days to allow complete polarisation (Natoli et al., 2012) and used when the trans-epithelial electrical resistance exceeded 900 $\Omega / \mathrm{cm}^{2}$ (Supplementary Figure S1A).

\section{Caco-2/Lactobacillus plantarum CUL66 co-incubation}

Overnight cultures of $L$. plantarum CUL66 were centrifuged $(1000 \times g, 10 \mathrm{~min})$ and the resultant pellet washed, by centrifugation and re-suspension, with antibiotic free DMEM supplemented with 4,500 mg/l glucose, $1 \%(\mathrm{v} / \mathrm{v})$ non-essential amino acids and $10 \mathrm{mM} \mathrm{N}$-(2-hydroxyethyl) piperazine-N'-(2-ethanesulfonic acid) (HEPES) and adjusted to $1 \times 10^{7}$ or $1 \times 10^{8} \mathrm{cfu} / \mathrm{ml}$ before application to Caco- 2 cells. For gene expression studies, $70 \mu \mathrm{g} / \mathrm{ml}$ cholesterol was added to all cells (including controls). Prior to co-incubation with L. plantarum CUL66, Caco-2 monolayers were washed three times with warm phosphate buffered saline (PBS) to remove all traces of antibiotic.

\section{Real-time quantitative polymerase chain reaction}

Caco-2 monolayers were washed three time with warm PBS and total RNA was isolated using Ribozol (Amresco LLC, Solon, OH, USA) and was reverse transcribed into cDNA using the High Capacity cDNA reverse transcription Kit (Life Technologies, Paisley, UK) in accordance with manufacturer protocols. RT-qPCR was performed on 10 ng cDNA using the iTag Universal SYBR Green SuperMix (Bio-Rad, Hemel Hempstead, $\mathrm{UK}$ ) in combination with $50 \mathrm{nM}$ each of the following gene specific primers (Eurofins Genomics, Ebersberg, Germany): 5'-TCTTCCCCTTCCTTGCCATT-3' and 5'-CGGCAGGGTAATTGTTGAGG-3' for NPC1L1; 5'-CCCAAGGGACTCCGGGGTCA-3' and 5'-GACCCATGGACCCTCCGGGG-3' for ABCG-5 (Alemany et al., 2013); 5'-GCCGCCCTCTTGTTCATG-3' and 5'-TAACATTTGGAGATGACATCCAGAA-3' for ABCG-8 (Kim et al., 2013); 5'-TTTCTCAGACAACACTTGACCAAGTA-3' and 5'-GGTTTTTGTGTAATGAGAGGTCTTTTAA-3' for ABCA-1 (Kim et al., 2013); 5'-GACCT T T C C A G A G C A A G C A C - 3, a n d 5' - T C A A C A AG AG C A T CGAGGG T-3' for 3-hydroxy-3-methylglutaryl-CoA reductase (HMGCR) and 5'-ACTCTTCCAGCCTTCCTTCC-3' and 5'-CGTACAGGTCTTTGCGGATG-3' for $\beta$-actin. Initial melting $\left(95^{\circ} \mathrm{C}\right.$ for $5 \mathrm{~min}$ ) followed by 40 cycles of melting $\left(94{ }^{\circ} \mathrm{C}\right.$ for $15 \mathrm{~s})$, annealing $\left(60^{\circ} \mathrm{C}\right.$ for $\left.15 \mathrm{~s}\right)$ and extension $\left(72{ }^{\circ} \mathrm{C}\right.$ for $30 \mathrm{~s}$ ) was performed using a CFX Connect ${ }^{\text {tw }}$ Real-Time Instrument (Bio-Rad) and fold changes in transcript level were determined using 2-( $\Delta \mathrm{C} t 1-\Delta \mathrm{C} t 2)$, where $\Delta C t$ represents the difference between the threshold cycle $(C T)$ for each target gene and $\beta$-Actin mRNA transcript levels. In order to eliminate amplification from contaminating genomic DNA all primer sets spanned an exon boundary and reverse transcriptase negative controls were included in each experiment. The amplicon sizes of each primer set were confirmed by agarose gel electrophoresis.

\section{Cholesterol uptake assay}

Cholesterol uptake was measured using a previously described method with minor adaptations (Huang et al., 2013). Polarised Caco- 2 cells were left untreated or incubated with $L$. plantarum CUL66 $\left(1 \times 10^{8} \mathrm{cfu} / \mathrm{ml}\right)$ for $6 \mathrm{~h}$. One hour before the end of incubation, $0.5 \mu \mathrm{Ci}$ $\left[4-{ }^{14} \mathrm{C}\right]$-cholesterol (Amersham Plc, Amersham, UK) was added to each well. At the end of the incubation, the cells were washed three times with cold DMEM to remove unincorporated radiolabelled cholesterol. Intracellular lipids were extracted by incubation with $1.0 \mathrm{ml}$ of ice cold hexane:isopropyl alcohol:water (3:2:0.1, v/v/v) for $30 \mathrm{~min}$ and radioactivity (disintegrations per min (DPM)) was measured using a liquid scintillation counter. Meanwhile, the remaining Caco-2 cell fraction was solubilised by incubation with $1 \mathrm{ml}$ of $0.2 \mathrm{M} \mathrm{NaOH}$ for $30 \mathrm{~min}$ at $37{ }^{\circ} \mathrm{C}$ and total cellular protein levels determined using the BCA protein assay kit (Life Technologies, Paisley, UK) in accordance with the manufacturer's instructions. DPM were normalised to protein levels and cholesterol uptake expressed as a percentage of the untreated control that has been arbitrarily set as $100 \%$. 


\section{Cholesterol efflux assay}

Cholesterol efflux was assessed using a method adapted from a previous study (Tachibana et al., 2007). Briefly, 21-day polarised Caco-2 cell monolayers grown on semipermeable transwell inserts were incubated with $\left[4-{ }^{14} \mathrm{C}\right]-$ cholesterol $(0.5 \mu \mathrm{Ci} /$ well $)$ in supplemented DMEM for $24 \mathrm{~h}$. Excess cholesterol was then removed with three rounds of washing with warm PBS. Apical intracellular cholesterol efflux was initiated by the addition of $1 \mathrm{mM}$ TDCA micelles alone or in combination with L. plantarum CUL66 $\left(1 \times 10^{8} \mathrm{cfu} / \mathrm{ml}\right)$ to the apical compartment while basolateral cholesterol efflux was simultaneously initiated by the addition of $10 \mu \mathrm{g} / \mathrm{ml}$ Apo-AI to the basolateral compartment. After $6 \mathrm{~h}$ incubation the media in both the apical and basolateral compartments was removed and retained for analysis. The remaining Caco- 2 cells were washed once with warm PBS and solubilised by incubation with $1 \mathrm{ml} 0.2 \mathrm{M} \mathrm{NaOH}$ for $30 \mathrm{~min}$ at $37^{\circ} \mathrm{C}$. DPM was measured in the apical, basolateral and cell fractions using a liquid scintillation counter and the percentage of intracellular cholesterol effluxed from the cells was determined by dividing the radioactivity of the apical media or basolateral media by the combined radioactivity in the apical media, basolateral media and cell fraction.

\section{Preparation of taurodeoxycholic acid micelles}

TDCA micelles were prepared as described elsewhere (Ikeda et al., 2002). Briefly, $0.6 \mathrm{mM}$ phosphatidylcholine, $1.0 \mathrm{mM}$ of oleic acid and $6.6 \mathrm{mM}$ of TDCA were thoroughly mixed in a glass vial and solvents evaporated under a flow of nitrogen. The resultant lipid film was then resolved in DMEM supplemented with 4,500 mg/l glucose, $1 \%(\mathrm{v} / \mathrm{v})$ non-essential amino acids and $10 \mathrm{mM}$ HEPES and filtered through a $0.4 \mu \mathrm{M}$ acetate filter before use.

\section{Statistical analysis}

All data are presented as the mean \pm standard deviation (SD) of the assigned number of independent experiments. Prior to significance testing, the normality of the data and the equality of group variance was confirmed using the Shapiro-Wilk and Levene's tests respectively. All data transformations are outlined in the figure legends. For single comparisons, values of $P$ were determined using Student's t-test. For multiple comparisons, values of $P$ were determined using one-way analysis of variance (ANOVA) with Tukey's post-hoc analysis. All statistical tests were performed using SPSS statistical software package version 22 (IBM, New York, NY, USA). Significance was defined when $P<0.05$.

\section{Results}

\section{BSH and cholesterol removal activity of Lactobacillus plantarum CUL66}

In three independent experiments a white precipitate was observed in the presence of $0.5 \%$ TDCA (Figure 1B), that was absent from the control (Figure 1A), indicating that $L$. plantarum CUL66 has BSH activity and this observation was confirmed on streak plates (Figures 1C and 1D). $L$. plantarum CUL66 also removed $28.3 \pm 15.2 \%$ of available cholesterol from MRS broth when compared to uninoculated control cholesterol levels $(P=0.009$, Figure $1 \mathrm{E})$ that equates to $3.35 \pm 1.98 \mathrm{mg}$ cholesterol per $\mathrm{g}$ of dry weight bacteria. The average $\mathrm{pH}$ of $18 \mathrm{~h}$ broths of L. plantarum CUL66 was 4.1.

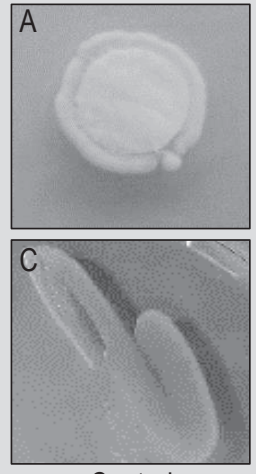

Control

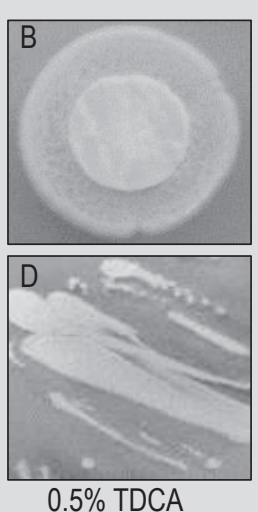

$0.5 \%$ TDCA

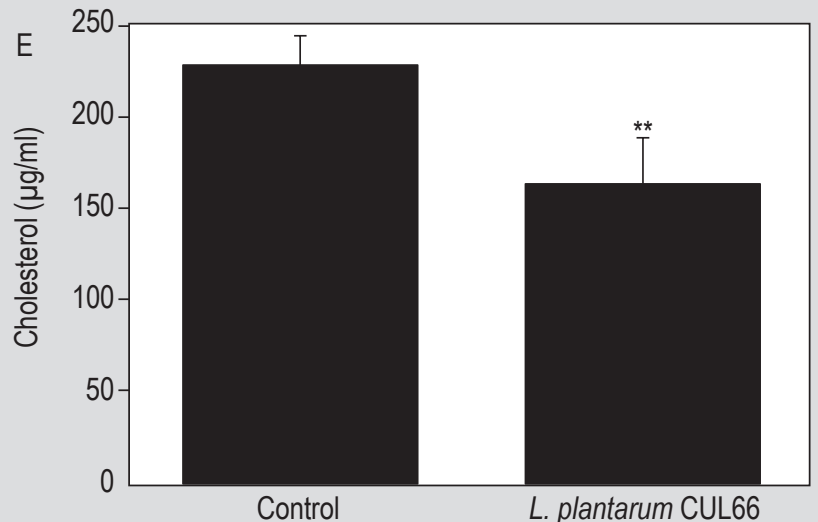

Control
L. plantarum CUL66

Figure 1. Bile salt hydrolase activity and ability to remove cholesterol from culture media by Lactobacillus plantarum CUL66. (AD) MRS agar plates (control, $A, C$ ) or MRS agar plates containing $0.5 \%$ taurodeoxycholic acid (TDCA) (B and D) were inoculated with $L$. plantarum CUL66 on filter discs ( $A$ and $B, n=3$ ) or as bacterial streaks ( $C$ and $D, n=1$ ) for $48 h$ under anaerobic conditions and assessed for the development of a white precipitate. (E) Cholesterol concentration in MRS broth containing $0.3 \%$ (w/v) ox-bile and $200 \mu \mathrm{g} / \mathrm{ml}$ cholesterol (control) or in MRS broth containing $0.3 \%$ (w/v) ox-bile and $200 \mu \mathrm{g} / \mathrm{ml}$ cholesterol inoculated with $L$. plantarum CUL66 for $18 \mathrm{~h}$ under anaerobic conditions. Data are presented as the mean \pm standard deviation from three independent experiments. Statistical analysis was performed using Student's t-test, where ${ }^{* *} P<0.001$. 
Expression of genes involved in Caco-2 cell cholesterol transport and metabolism

As shown in Figure 2A, co-incubation of polarised Caco-2 cells with cholesterol $(70 \mu \mathrm{g} / \mathrm{ml})$ and L. plantarum CUL66 $\left(1 \times 10^{8} \mathrm{cfu} / \mathrm{ml}\right)$ resulted in a $19 \%(P=0.015)$ reduction in the expression of NPC1L1 when compared to cells treated with cholesterol alone (Control). Under the same experimental conditions the expression of ABCG-5 (Figure 2B) and ABCG-8 (Figure 2C) were significantly increased by 3.19fold $(P<0.001)$ and 2.18-fold $(P=0.004)$, respectively, while ABCA-1 transcript levels (Figure 2 D) were significantly reduced $(45 \% ; P<0.001)$. Analysis of HMGCR transcript levels (Figure $2 \mathrm{E})$ revealed a $63 \%(P=0.039)$ induction in response to $1 \times 10^{8} \mathrm{cfu} / \mathrm{ml}$ L. plantarum CUL66 when compared to the control. No significant changes in expression were observed for any of the genes tested in response to $1 \times 10^{7} \mathrm{cfu} / \mathrm{ml}$ L. plantarum CUL66 suggesting that a threshold number of viable organisms is required. There were indications of a dose-response in expression for most of the genes at the two doses tested. The viability of both Caco- 2 cells and L. plantarum CUL66 were retained throughout the duration of the experiment (Supplementary Figures S1B and S1C, respectively). The average $\mathrm{pH}$ of the $6 \mathrm{~h}$ co-culture supernatants was 6.3 .

\section{Cholesterol uptake by Caco- 2 cells inhibition by Lactobacillus plantarum CUL66}

Reduced transcript levels of NPC1L1 (Figure 2A) suggest that L. plantarum CUL66 may have the ability to inhibit the uptake of cholesterol by intestinal epithelial cells. Polarised
A

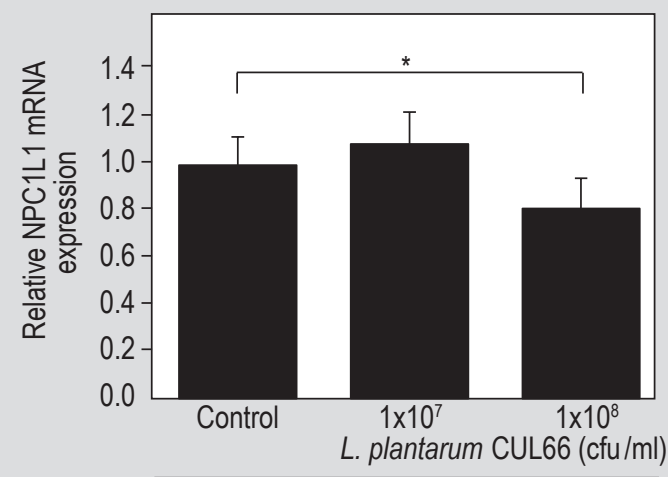

C
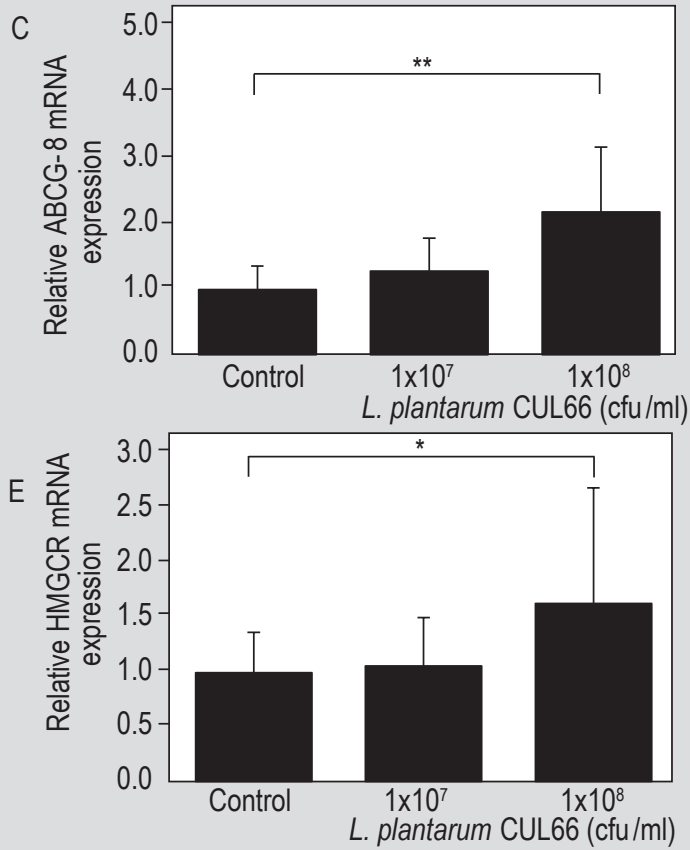

B
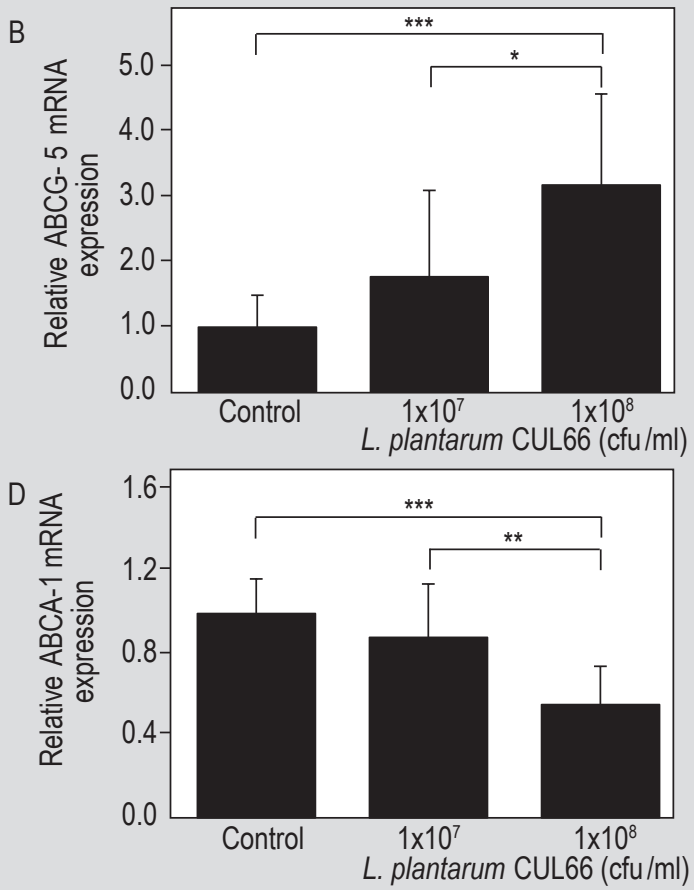

Figure 2. Gene transcript levels of (A) Niemann-Pick C1-like 1 (NPC1L1), (B) ATP-binding cassette sub-family G member (ABCG)5, (C) ABCG-8, (D) ATP-binding cassette transporter-1 (ABCA-1) and (E) 3-hydroxy-3-methylglutaryl-CoA reductase (HMGCR) in polarised Caco-2 cells treated with $70 \mu \mathrm{g} / \mathrm{ml}$ cholesterol (Control) or cholesterol $(70 \mu \mathrm{g} / \mathrm{ml}$ ) and Lactobacillus plantarum CUL66 $\left(1 \times 10^{7}\right.$ or $\left.1 \times 10^{8} \mathrm{cfu} / \mathrm{ml}\right)$ for $6 \mathrm{~h}$. Data are presented as the mean \pm standard deviation from at least four independent experiments. Statistical analysis was performed using a one-way ANOVA with Tukey's post-hoc analysis (on log-transformed data for ABCG-5, ABCG-8 and HMGCR), where ${ }^{*} P<0.05$, ${ }^{* *} P<0.01$ and ${ }^{* * *} P<0.001$. 
Caco-2 cells were incubated with L. plantarum CUL66 for $5 \mathrm{~h}$ before radiolabelled cholesterol was added for $1 \mathrm{~h}$. As shown in Figure 3, incubation with L. plantarum CUL66 significantly reduced the uptake of radiolabelled cholesterol by Caco-2 cells by $16.52 \%(P<0.001)$ when compared to the untreated control. The magnitude of reduction of uptake (16\%) is in line with the reduced gene expression of NPC1L1 (19\%).

\section{Inhibition of cholesterol efflux into Caco-2 basolateral compartments by Lactobacillus plantarum CUL66}

Caco-2 cells were grown on semi-permeable transwell inserts that allowed assessment of intracellular cholesterol efflux into the apical or basolateral compartments. The inoculation of polarised Caco-2 cells with L. plantarum CUL66 $\left(1 \times 10^{8} \mathrm{cfu} / \mathrm{ml}\right)$ had no significant effect on the apical efflux of cholesterol (Figure 4A), while a significant $51.97 \%$ $(P<0.001)$ reduction in the proportion of cholesterol moved to the basolateral compartment was observed in response to L. plantarum CUL66 when compared to untreated cells (Figure 4B). This is in line with the observed reduction in gene expression of ABCA-1 (45\%).

\section{Discussion}

These studies have focused on the impact of $L$. plantarum CUL66 (NCIMB 30280) on cholesterol uptake/metabolism by Caco-2 epithelial cells. L. plantarum CUL66 was shown to remove nearly $30 \%$ of the cholesterol component from culture medium over a $24 \mathrm{~h}$ incubation period and was found to possess significant BSH activity. After 5 h incubation with L. plantarum CUL66, uptake of radiolabelled cholesterol by Caco- 2 cells was significantly

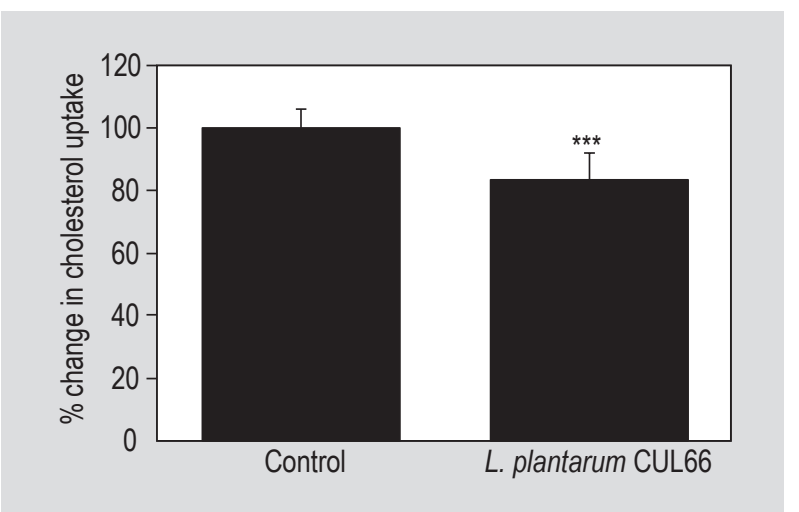

Figure 3. Lactobacillus plantarum CUL66 inhibits the uptake of cholesterol by polarised Caco-2 cells. Polarised Caco-2 cells were untreated (Control) or incubated with L. plantarum CUL66 $\left(1 \times 10^{8} \mathrm{cfu} / \mathrm{ml}\right)$. Intracellular radioactivity was normalised to total protein content and presented as a percentage of the control that has been arbitrarily assigned as $100 \%$. Data are presented as the mean \pm standard deviation from three independent experiments. Statistical analysis was performed using Student's t-test, where *** $P<0.001$. lower than in cells exposed to radiolabelled cholesterol without prior exposure to L. plantarum CUL66. L. plantarum CUL66 caused a significant decrease in NPC1L1 mRNA expression by the epithelial cells which is likely to contribute to these observations. The expression of both ABCG-5 and ABCG-8 was increased in Caco- 2 cells in the presence of L. plantarum CUL66 which would favour the apical efflux of cholesterol from the intestinal epithelium into the intestinal lumen, however, no differences in the amount of radiolabelled cholesterol were observed in the apical compartment of the transwell model after 6 h exposure to L. plantarum CUL66. In contrast, a clear reduction in the amount of cholesterol efflux from Caco2 cells into the basolateral compartment was observed after $6 \mathrm{~h}$ exposure to L. plantarum CUL66 and occurred alongside reduced expression of cholesterol transporter ABCA-1. Significantly elevated transcript levels of HMGCR; the rate limiting enzyme during the de novo synthesis of cholesterol, were also observed in Caco- 2 cells exposed to L. plantarum CUL66.

A

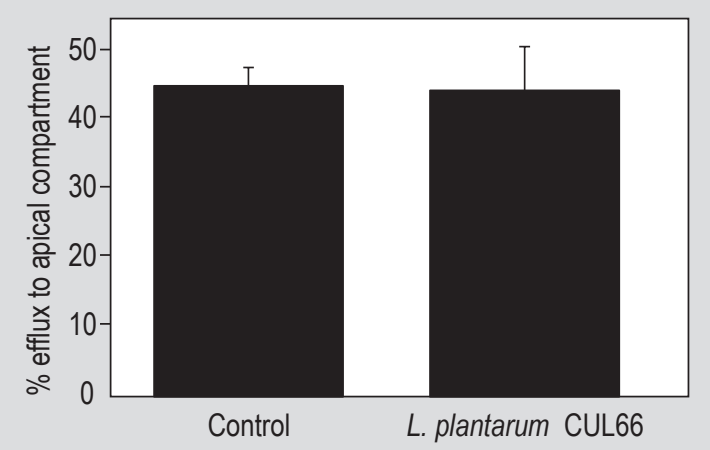

B

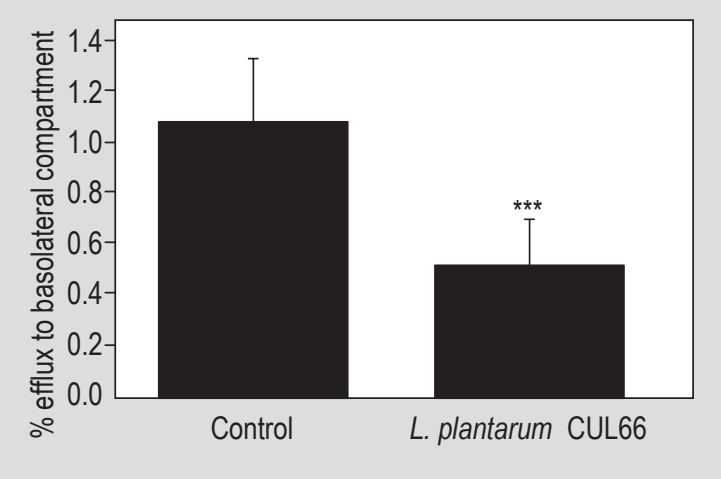

Figure 4. Influence of Lactobacillus plantarum CUL66 on the efflux of $(A)$ intracellular radiolabelled cholesterol to taurodeoxycholic acid micelles in the apical compartment and $(B)$ apolipoprotrein-Al in the basolateral compartment of polarised Caco-2 cells. Polarised Caco- 2 cells were untreated (Control) or incubated with L. plantarum CUL66 ( $1 \times 10^{8} \mathrm{cfu} /$ $\mathrm{ml})$. Data are presented as mean \pm standard deviation from at least three independent experiments. Statistical analysis was performed using Student's t-test, where ${ }^{* * *} P<0.001$. 
The cholesterol lowering activities of probiotic bacteria have been demonstrated and a diversity of bacterial strains have been identified (Fuentes et al., 2013; Guo et al., 2011; Sun and Buys, 2015). BSH activity has been observed in various strains of L. plantarum and cholesterol lowering activity has been identified in a diversity of probiotic organisms at levels comparable with L. plantarum CUL66 (Gorenjak et al., 2014; Pereira and Gibson, 2002a; Tanaka et al., 1999; Tomaro-Duchesneau et al., 2014). Variations in the levels of cholesterol uptake were observed with L. plantarum CUL66 as was seen by Pereira and Gibson for a range of bacterial strains (Pereira and Gibson, 2002a). In our L. plantarum CUL66 study the pH was not controlled (final pH of 4.1) and it has been found that cholesterol can precipitate out of culture media with conjugated bile salts when the $\mathrm{pH}$ drops below 5.5 (Klaver and Van der Meer, 1993) which could have potentially made some contribution to the outcome. It is generally accepted that in vitro cholesterol assimilation can be used as an important predictor of in vivo cholesterol lowering ability although Madani et al. (2013) found little correlation to support this assumption. However, work by Mahenthiralingam et al. (2009) has clearly demonstrated the ability of lactic acid bacteria to survive transit through the human gut suggesting that L. plantarum CUL66 could be expected to be viable in the intestine, and non-viable bacteria have been shown to take up cholesterol (Liong and Shah, 2005; Wang et al., 2014; Zeng et al., 2010) which may suggest the involvement of other probiotic-mediated cholesterol lowering mechanisms.

To this end, we have shown that L. plantarum CUL66 can inhibit NPC1L1 gene expression and reduce cholesterol uptake by polarised Caco-2 cells. These observations are corroborated by in vitro and in vivo studies that report similar changes in NPC1L1 gene expression in response to L. plantarum strains PCS20, PCS26, NR74 or Lp27 (Gorenjak et al., 2014; Huang et al., 2013; Yoon et al., 2013) that manifest as reduced cholesterol uptake by NR74 treated Caco-2 cells (Yoon et al., 2013) and reduced plasma cholesterol levels in Lp24 fed hypercholesteremic rats (Huang et al., 2013). The ability of L. plantarum CUL66 to regulate cholesterol transport by Caco- 2 cells may also extend to the regulation of cholesterol efflux in light of the increased levels of ABCG-5 and ABCG-8 transcripts observed in our system. Similar observations have been made in response to L. plantarum strains PCS20, PSC26 or NR74 in numerous intestinal epithelial cell types (Gorenjak et al., 2014; Yoon et al., 2011) that, in the case of L. plantarum NR74, correlated with a clear increase in apical cholesterol excretion from non-polarised Caco-2 cells (Yoon et al., 2011). While we did not observe similar changes in cholesterol efflux into the apical compartment of our transwell model we did observe clear reductions in ABCA-1 expression and cholesterol efflux from Caco-2 cells to the basolateral compartment in response to $L$. plantarum CUL66 and are therefore the first to suggest that the ABCA-1/ApoA-I pathway can be regulated by such bacteria in enterocytes.

In addition, we also observed an increase in the expression of HMGCR in response to L. plantarum CUL66 that contrast the findings of another study showing reduced HMGCR expression in Caco-2 cells exposed to a mixed culture of Escherichia coli 6-1 and L. plantarum (ATCC 202195) (Panigrahi et al., 2007). Interestingly, increased intestinal expression of HMGCR has also been observed in numerous in vivo experiments examining the molecular event associated with ezetimibe-mediated inhibition of cholesterol absorption (Catry et al., 2015; Engelking et al., 2012; Repa et al., 2005; Telford et al., 2007; Valasek et al., 2008) and is thought to form part of a sterol regulatory element-binding protein-dependent compensatory mechanism by the host to maintain cholesterol levels (Catry et al., 2015; Engelking et al., 2012; Telford et al., 2007).

In summary, this study reports the ability of $L$. plantarum CUL66 to beneficially regulate multiple in vitro models associated with intestinal cholesterol absorption and therefore implicates it as a holistic approach to reduce serum cholesterol levels via the intestine. To date, no other studies have reported the ability of L. plantarum CUL66 to deconjugate bile salts and assimilate cholesterol in vitro, nor its ability to beneficially regulate cholesterol transport across polarised Caco- 2 cells through the coordinated inhibition of intestinal cholesterol uptake and basolateral cholesterol efflux. This study provides adequate evidence of efficacy for the inclusion of L. plantarum CUL66 in future in vivo studies.

\section{Supplementary material}

Supplementary material can be found online at http:// dx.doi.org/10.3920/BM2015.0146.

Figure S1. Trans-epithelial electrical resistance of cultured Caco- 2 cells and the cell viability under experimental conditions.

\section{Conflict of interest}

This study was supported by Cultech Ltd, Port Talbot, UK. DRM, DLC, IG and SFP are employees of Cultech Ltd. JWEM is a PhD student funded by a joint studentship from the School of Biosciences, Cardiff University and Cultech Ltd.

\section{Acknowledgements}

The authors would like to acknowledge Annalise S. Hooper, Kathryn A. Turner and Hedda L. Köhling of Cultech Limited for their technical assistance during the study. 


\section{References}

Alemany, L., Laparra, J.M., Barberá, R. and Alegría, A., 2013. Relative expression of cholesterol transport-related proteins and inflammation markers through the induction of 7-ketosterolmediated stress in Caco-2 cells. Food and Chemical Toxicology 56: 247-253.

Altmann, S.W., Davis, H.R., Zhu, L.J., Yao, X., Hoos, L.M., Tetzloff, G., Iyer, S.P., Maguire, M., Golovko, A., Zeng, M., Wang, L., Murgolo, N. and Graziano, M.P., 2004. Niemann-Pick C1 Like 1 protein is critical for intestinal cholesterol absorption. Science 303: 1201-1204.

Banach, M., Rizzo, M., Toth, P.P., Farnier, M., Davidson, M.H., AlRasadi, K., Aronow, W.S., Athyros, V., Djuric, D.M., Ezhov, M.V., Greenfield, R.S., Hovingh, G.K., Kostner, K., Serban, C., Lighezan, D., Fras, Z., Moriarty, P.M., Muntner, P., Goudev, A., Ceska, R., Nicholls, S.J., Broncel, M., Nikolic, D., Pella, D., Puri, R., Rysz, J., Wong, N.D., Bajnok, L., Jones, S.R., Ray, K.K. and Mikhailidis, D.P., 2015. Statin intolerance - an attempt at a unified definition. Position paper from an International Lipid Expert Panel. Archives of Medical Science 11: 1-23.

Berge, K.E., Tian, H., Graf, G.A., Yu, L., Grishin, N.V., Schultz, J., Kwiterovich, P., Shan, B., Barnes, R. and Hobbs, H.H., 2000. Accumulation of dietary cholesterol in sitosterolemia caused by mutations in adjacent ABC transporters. Science 290: 1771-1775.

Buckley, M.L. and Ramji, D.P., 2015. The influence of dysfunctional signaling and lipid homeostasis in mediating the inflammatory responses during atherosclerosis. Biochimica et Biophysica Acta 1852: $1498-1510$

Cannon, C.P., Blazing, M.A., Giugliano, R.P., McCagg, A., White, J.A., Theroux, P., Darius, H., Lewis, B.S., Ophuis, T.O., Jukema, J.W., De Ferrari, G.M., Ruzyllo, W., De Lucca, P., Im, K., Bohula, E.A., Reist, C., Wiviott, S.D., Tershakovec, A.M., Musliner, T.A., Braunwald, E., Califf, R.M. and the Improve-It Investigators, 2015. Ezetimibe Added to statin therapy after acute coronary syndromes. New England Journal of Medicine 372: 2387-2397.

Catry, E., Pachikian, B.D., Salazar, N., Neyrinck, A.M., Cani, P.D. and Delzenne, N.M., 2015. Ezetimibe and simvastatin modulate gut microbiota and expression of genes related to cholesterol metabolism. Life Sciences 132: 77-84.

Christie, C., 2015. Proposed Dietary Guidelines 2015 and implications for cardiovascular disease and diabetes. Journal of Cardiovascular Nursing 30: 375-378

Davis, H.R., Hoos, L.M., Tetzloff, G., Maguire, M., Zhu, L.J., Graziano, M.P. and Altmann, S.W., 2007. Deficiency of Niemann-Pick C1 Like 1 prevents atherosclerosis in $\mathrm{ApoE}^{-/-}$mice. Arteriosclerosis, Thrombosis and Vascular Biology 27: 841-849.

Drobnik, W., Lindenthal, B., Lieser, B., Ritter, M., Christiansen Weber, T., Liebisch, G., Giesa, U., Igel, M., Borsukova, H., Büchler, C., Fung-Leung, W.P., Von Bergmann, K. and Schmitz, G., 2001. ATPbinding cassette transporter A1 (ABCA1) affects total body sterol metabolism. Gastroenterology 120: 1203-1211.

Engelking, L..., McFarlane, M.R., Li, C.K. and Liang, G., 2012. Blockade of cholesterol absorption by ezetimibe reveals a complex homeostatic network in enterocytes. Journal of Lipid Research 53: 1359-1368.
Food and Agriculture Organization of the United nations/World Health Organization (FAO/WHO), 2006. Probiotics in food. Health and nutritional properties and guidelines for evaluation. FAO Food and Nutritional paper no. 85. FAO, Rome, Italy. Available at: http:// tinyurl.com/8bccc3r.

Fuentes, M.C., Lajo, T., Carrión, J.M. and Cuñé, J., 2013. Cholesterollowering efficacy of Lactobacillus plantarum CECT 7527, 7528 and 7529 in hypercholesterolaemic adults. British Journal of Nutrition 109: 1866-1872.

Gorenjak, M., Gradišnik, L., Trapečar, M., Pistello, M., Kozmus, C.P., Škorjanc, D., Skok, P., Langerholc, T. and Cencič, A., 2014. Improvement of lipid profile by probiotic/protective cultures: study in a non-c arcinogenic small intestinal cell model. New Microbiologica 37: 51-64.

Guo, Z., Liu, X.M., Zhang, Q.X., Shen, Z., Tian, F.W., Zhang, H., Sun, Z.H., Zhang, H.P. and Chen, W., 2011. Influence of consumption of probiotics on the plasma lipid profile: a meta-analysis of randomised controlled trials. Nutrition, Metabolism, and Cardiovascular Diseases 21: 844-850.

Huang, Y. and Zheng, Y., 2010. The probiotic Lactobacillus acidophilus reduces cholesterol absorption through the down-regulation of Niemann-Pick C1-like 1 in Caco-2 cells. British Journal of Nutrition 103: 473-478.

Huang, Y., Wang, J., Cheng, Y. and Zheng, Y., 2010. The hypocholesterolaemic effects of Lactobacillus acidophilus American type culture collection 4356 in rats are mediated by the downregulation of Niemann-Pick C1-like 1. British Journal of Nutrition 104: 807-812.

Huang, Y., Wu, F., Wang, X., Sui, Y., Yang, L. and Wang, J., 2013. Characterization of Lactobacillus plantarum Lp27 isolated from Tibetan kefir grains: a potential probiotic bacterium with cholesterol-lowering effects. Journal of Dairy Science 96: 2816-2825.

Ikeda, I., Matsuoka, R., Hamada, T., Mitsui, K., Imabayashi, S., Uchino, A., Sato, M., Kuwano, E., Itamura, T., Yamada, K., Tanaka, K. and Imaizumi, K., 2002. Cholesterol esterase accelerates intestinal cholesterol absorption. Biochimica et Biophysica Acta 1571: 34-44.

Kim, B., Park, Y., Wegner, C.J., Bolling, B.W. and Lee, J., 2013. Polyphenol-rich black chokeberry (Aronia melanocarpa) extract regulates the expression of genes critical for intestinal cholesterol flux in Caco-2 cells. Journal of Nutritional Biochemistry 24: 1564-1570.

Klaver, F.A. and van der Meer, R., 1993. The assumed assimilation of cholesterol by lactobacilli and Bifidobacterium bifidum is due to their bile salt-deconjugating activity. Applied and Environmental Microbiology 59: 1120-1124.

Kumar, M., Nagpal, R., Kumar, R., Hemalatha, R., Verma, V., Kumar, A., Chakraborty, C., Singh, B., Marotta, F., Jain, S. and Yadav, H., 2012. Cholesterol-lowering probiotics as potential biotherapeutics for metabolic diseases. Experimental Diabetes Research 2012: 902917.

Liong, M.T. and Shah, N.P., 2005. Acid and bile tolerance and cholesterol removal ability of lactobacilli strains. Journal of Dairy Science 88: 55-66.

Madani, G., Mirlohi, M., Yahay, M. and Hassanzadeh, A., 2013. How much in vitro cholesterol reducing activity of lactobacilli predicts their in vivo cholesterol function? International Journal of Preventive Medicine 4: 404-413. 
Mahenthiralingam, E., Marchbank, A., Drevinek, P., Garaiova, I. and Plummer, S., 2009. Use of colony-based bacterial strain typing for tracking the fate of Lactobacillus strains during human consumption. BMC Microbiology 9: 251.

Mann, G.V., 1974. Studies of a surfactant and cholesteremia in the Maasai. American Journal of Clinical Nutrition 27: 464-469.

McLaren, J.E., Michael, D.R., Ashlin, T.G. and Ramji, D.P., 2011. Cytokines, macrophage lipid metabolism and foam cells: implications for cardiovascular disease therapy. Progress in Lipid Research 50: 331-347.

Michael, D.R., Ashlin, T.G., Buckley, M.L. and Ramji, D.P., 2012. Macrophages, lipid metabolism and gene expression in atherogenesis: a therapeutic target of the future? Clinical Lipidology 7: 37-48.

Natoli, M., Leoni, B.D., D’Agnano, I., Zucco, F. and Felsani, A., 2012. Good Caco-2 cell culture practices. Toxicology In Vitro 26: 1243-1246.

Ohama, T., Hirano, K., Zhang, Z., Aoki, R., Tsujii, K., NakagawaToyama, Y., Tsukamoto, K., Ikegami, C., Matsuyama, A., Ishigami, M., Sakai, N., Hiraoka, H., Ueda, K., Yamashita, S. and Matsuzawa, Y., 2002. Dominant expression of ATP-binding cassette transporter-1 on basolateral surface of Caco-2 cells stimulated by LXR/RXR ligands. Biochemical and Biophysical Research Communications 296: 625-630.

Panigrahi, P., Braileanu, G.T., Chen, H. and Stine, O.C., 2007. Probiotic bacteria change Escherichia coli-induced gene expression in cultured colonocytes: Implications in intestinal pathophysiology. World Journal of Gastroenterology 13: 6370-6378.

Pereira, D.I. and Gibson, G.R., 2002a. Cholesterol assimilation by lactic acid bacteria and bifidobacteria isolated from the human gut. Applied and Environmental Microbiology 68: 4689-4693.

Pereira, D.I. and Gibson, G.R., 2002b. Effects of consumption of probiotics and prebiotics on serum lipid levels in humans. Critical Reviews in Biochemistry and Molecular Biology 37: 259-281.

Repa, J.J., Turley, S.D., Quan, G. and Dietschy, J.M., 2005. Delineation of molecular changes in intrahepatic cholesterol metabolism resulting from diminished cholesterol absorption. Journal of Lipid Research 46: 779-789.

Rerksuppaphol, S. and Rerksuppaphol, L., 2015. A randomized doubleblind controlled trial of Lactobacillus acidophilus plus Bifidobacterium bifidum versus placebo in patients with hypercholesterolemia. Journal of Clinical and Diagnostic Research 9: KC01-04.

Rudel, L.L. and Morris, M.D., 1973. Determination of cholesterol using o-phthalaldehyde. Journal of Lipid Research 14: 364-366.

Sudhop, T., Lütjohann, D., Kodal, A., Igel, M., Tribble, D.L., Shah, S., Perevozskaya, I. and von Bergmann, K., 2002. Inhibition of intestinal cholesterol absorption by ezetimibe in humans. Circulation 106: 1943-1948.

Sun, J. and Buys, N., 2015. Effects of probiotics consumption on lowering lipids and CVD risk factors: a systematic review and meta-analysis of randomized controlled trials. Annals of Medicine 47: 430-440.

Tachibana, S., Hirano, M., Hirata, T., Matsuo, M., Ikeda, I., Ueda, K. and Sato, R., 2007. Cholesterol and plant sterol efflux from cultured intestinal epithelial cells is mediated by ATP-binding cassette transporters. Bioscience Biotechnology and Biochemistry 71: 1886-1895.
Tanaka, H., Doesburg, K., Iwasaki, T. and Mierau, I., 1999. Screening of lactic acid bacteria for bile salt hydrolase activity. Journal of Dairy Science 82: 2530-2535.

Taylor, F., Huffman, M.D., Macedo, A.F., Moore, T.H., Burke, M., Davey Smith, G., Ward, K. and Ebrahim, S., 2013. Statins for the primary prevention of cardiovascular disease. Cochrane Database of Systematic Reviews 1: CD004816.

Telford, D.E., Sutherland, B.G., Edwards, J.Y., Andrews, J.D., Barrett, P.H. and Huff, M.W., 2007. The molecular mechanisms underlying the reduction of LDL apoB-100 by ezetimibe plus simvastatin. Journal of Lipid Research 48: 699-708.

Tomaro-Duchesneau, C., Jones, M.L., Shah, D., Jain, P., Saha, S. and Prakash, S., 2014. Cholesterol assimilation by Lactobacillus probiotic bacteria: an in vitro investigation. Biomed Research International 2014: 380316.

Townsend, N., Wickramasinghe, K., Bhatnagar, P., Smolina, K., Nichols, M., Luengo-Fernandez, R. and Rayner, M., 2012. Coronary heart disease statistics 2012 edition. British Heart Foundation, London, UK.

Valasek, M.A., Repa, J.J., Quan, G., Dietschy, J.M. and Turley, S.D., 2008. Inhibiting intestinal NPC1L1 activity prevents diet-induced increase in biliary cholesterol in Golden Syrian hamsters. American Journal of Physiology - Gastrointestinal and Liver Physiology 295: G813-G822.

Vrins, C., Vink, E., Vandenberghe, K.E., Frijters, R., Seppen, J. and Groen, A.K., 2007. The sterol transporting heterodimer ABCG5/ ABCG8 requires bile salts to mediate cholesterol efflux. FEBS Letters 581: 4616-4620.

Wang, S.C., Chang, C.K., Chan, S.C., Shieh, J.S., Chiu, C.K. and Duh, P.D., 2014. Effects of lactic acid bacteria isolated from fermented mustard on lowering cholesterol. Asian Pacific Journal of Tropical Biomedicine 4: 523-528.

World Health Organization (WHO), 2015. Cardiovascular diseases (CVDs). Fact sheet 317. WHO, Geneva, Switzerland. Available at: http://tinyurl.com/br89ujf.

Yoon, H.S., Ju, J.H., Kim, H., Lee, J., Park, H.J., Ji, Y., Shin, H.K., Do, M.S., Lee, J.M. and Holzapfel, W., 2011. Lactobacillus rhamnousus BFE 5264 and Lactobacillus plantarum NR74 promote cholesterol excretion through the up-regulation of ABCG5/8 in caco-2 cells. Probiotics and Antimicrobial Proteins 3: 194-203.

Yoon, H.S., Ju, J.H., Kim, H.N., Park, H.J., Ji, Y., Lee, J.E., Shin, H.K., Do, M.S. and Holzapfel, W., 2013. Reduction in cholesterol absorption in Caco-2 cells through the down-regulation of Niemann-Pick C1like 1 by the putative probiotic strains Lactobacillus rhamnosus BFE5264 and Lactobacillus plantarum NR74 from fermented foods. International Journal of Food Sciences and Nutrition 64: 44-52.

Yu, L., Hammer, R.E., Li-Hawkins, J., Von Bergmann, K., Lutjohann, D., Cohen, J.C. and Hobbs, H.H., 2002. Disruption of Abcg5 and Abcg8 in mice reveals their crucial role in biliary cholesterol secretion. Proceedings of the National Academy of Sciences of the USA 99: 16237-16242.

Zeng, X.Q., Pan, D.D. and Guo, Y.X., 2010. The probiotic properties of Lactobacillus buchneri P2. Journal of Applied Microbiology 108: 2059-2066. 
